\title{
HANDLING DATABASES IN SEARCH FOR MICROQUASARS
}

\author{
Pedro Luis Luque-Escamilla * \\ FAEG Group-Universidad de Jaén \\ E-mail: peter@ujaen.es
}

\section{Josep Martí; Jorge Ariel Combi; J.R. Sánchez Sutil; Álvaro Muñoz-Arjonilla}

FAEG Group-Universidad de Jaén

\section{J.F. Albacete-Colombo}

Centro Universitario Regional Zona Atlántica (CURZA). Universidad Nacional del COMAHUE

\begin{abstract}
Microquasars are X-ray binary systems capable of generate relativistic radio jets, and may be used as a benchmark for accretion/ejection models due to its scaled-down response times. The search for such objects is a difficult task, and up to now only about 15 microquasars have been confirmed. The cross-identification between catalogues of different wavelengths appeared as a promising approach to find microquasar candidates but success has been limited so far [7|[9]. Recently, our group used the new MAGPIS survey data to find coincidences with the Luminous Star Catalogue which derives in the identification of LS 5007 as a microquasar candidate, although follow up VLA radio observations of the source point more to a stellar wind as the cause of the radio and X-ray emissions. In order to avoid false detections, a more stringent selection criteria for the cross identifications in the databases have derived in an improved approach from which 3 objects have been proposed as likely galactic high mass X-ray binary systems and microquasar candidates ([1]).
\end{abstract}

VII Microquasar Workshop: Microquasars and Beyond

September 1 - 5, 2008

Foca, Izmir, Turkey

*J.A.C. is a researcher of the programme Ramón y Cajal funded jointly by the Spanish Ministerio de Educación y Ciencia and Universidad de Jaén. The authors also acknowledge support by DGI of the Spanish Ministerio de Educación y Ciencia under grants AYA2007-68034-C03- 02, FEDER funds and Plan Andaluz de Investigación Desarrollo e Innovación (PAIDI) of Junta de Andalucía as research group FQM322. J.F.A.C. is researcher of the Consejo Nacional de Investigaciones Científicas y Tecnológicas (CONICET), Argentina. This research has also made use of the NASA/IPAC Infrared Science Archive, which is operated by the Jet Propulsion Laboratory, California Institute of Technology, under contract with the National Aeronautics and Space Administration. In addition, this research makes use of data products from the Two Micron All Sky Survey, which is a joint project of the University of Massachusetts and the Infrared Processing and Analysis Center/California Institute of Technology, funded by the National Aeronautics and Space Administration and the National Science Foundation. 


\section{Introduction}

Microquasars are X-ray binary systems with properties that resemble those of quasars. They are composed of a compact object (black hole or neutron star) that is accreting matter from its companion star. Because of the deep gravity well, a huge amount of energy can be released in $\mathrm{X}$-rays from the accretion disk. Relativistic jets are also formed which radiate a broad band nonthermal spectrum from radio to even very high-energy gamma-rays. Microquasars undergo strong flares from time to time due to the dynamics of the jet formation. When the jets interact with the interstellar medium (ISM), extended bow shocks may appear as it has been recently illustrated in the Cygnus X-1 case [2]. The situation eventually becomes analogous to the case of lobes from radio galaxies (see, for instance, $[4]$ and references therein).

Despite their interesting nature, microquasars seem to be rare objects in our Galaxy. Only 15 are confirmed (6 high-mass X-ray binaries, 9 low-mass X-ray binaries)[8]. Finding new candidates is not an easy task. One approach is based on cross-identifications between radio, infrared and X-ray catalogues. This methodology originally led to the identification of LS 5039 as a possible microquasar system, responsible for an EGRET source of high-energy gamma-rays [6]. Cross-identification among different wavelength catalogues is based on the different signatures a microquasar exhibits. Radio emission may be due to incoherent synchrotron emission in the relativistic jet, while X-rays or even more energetic radiation may reveal accretion/ejection processes. Emission in ultraviolet and infrared may be due to the star companion. Thus, applying restrictive selection criteria some candidates may be proposed. However, this is not always successful [7], [9], mainly because the ROSAT X-ray data used in previous searches was not sensitive to energetic enough photons.

\section{Improving the database cross identification}

Recently, our group performed a new cross identification from the new Multi-Array Galactic Plane Imaging Survey (MAGPIS) data [11], [3]together with ROSAT, XMM and the Catalogue of Luminous Stars in the Southern Milky Way [10]. The coincidences found led to the identification of the luminous star LS 5007 as a possible microquasar candidate (Fig 1), although follow-up VLA

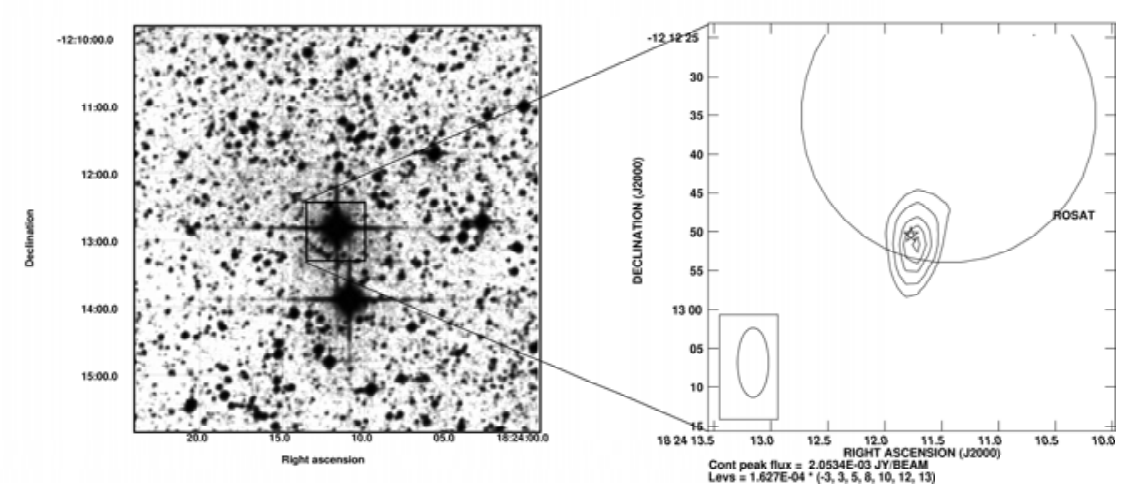

Figure 1: LS 5007 in optical (DSS2 Red),radio (VLA) and X-rays (ROSAT), from left to right. 


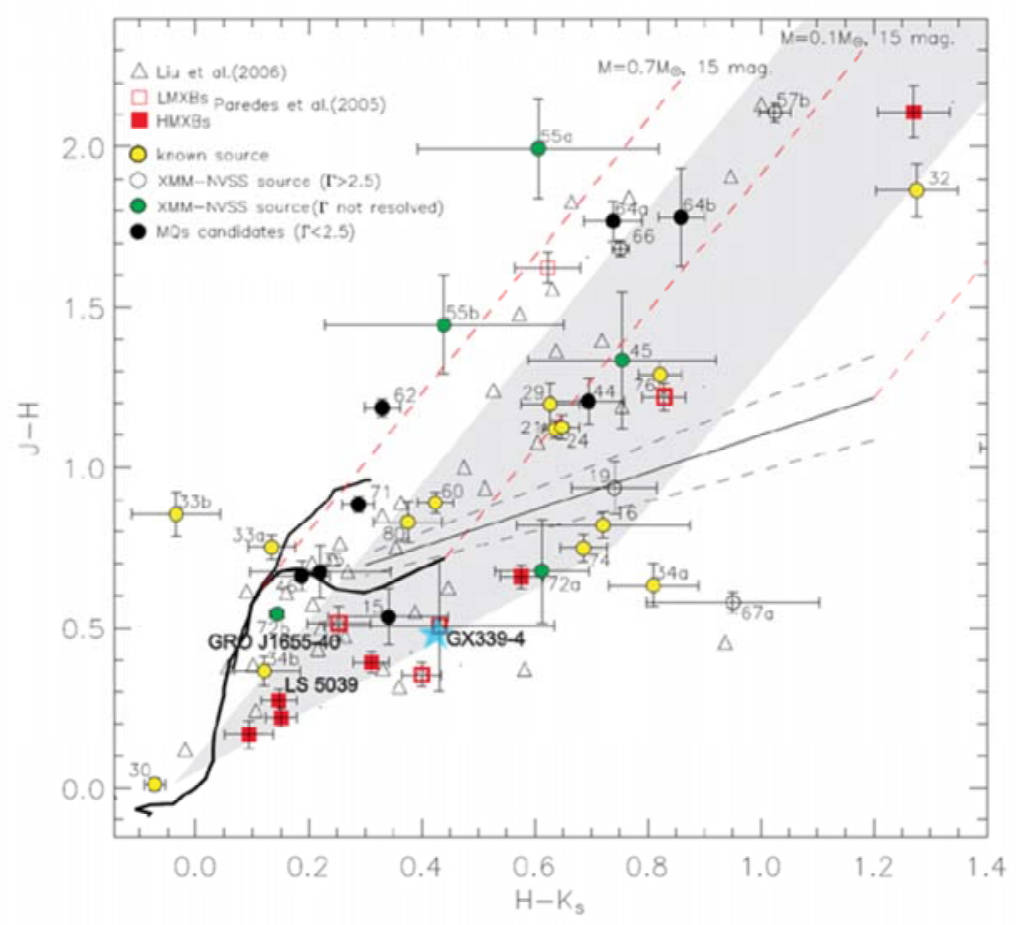

Figure 2: A near infrared color-color diagram showing the position of known microquasars (red) and candidates (black)[1]. The grey region is the proposed for possible microquasars based on the behaviour of known microquasars with high mass. LS 5007 appears as a blue star.

radio observations of this source are more suggestive of a stellar wind as the cause of the radio and $\mathrm{X}$-ray emissions, mainly due to a radio spectral index close to the canonical value of 0.6 for this scenario and its reltively low X-ray luminosity. However, its position in a near infrared color-color graph is within the loci for microquasar candidates (Fig 2) [1]. Its hardness ratio obtained from ROSAT-PSPC is 0.47 in $[0.2,1\rceil \mathrm{keV}$ soft band, and 0.94 in $\lceil 1,2.4\rceil \mathrm{keV}$ band.

In order to improve the efficiency of the cross identifications, the search strategy has been improved by using a new very stringent selection criteria and it has being applied to modern and more sensitive multiwavelength data in a recent paper [1]. First we search for positional coincidences with galactic latitudes $|b| \leq 10^{\circ}$. From these sources, we seek for infrared counterparts in the 2MASS catalogue for the non-identified ones. When available, the hardness ratio was used to assess the possible low-mass or high-mass X-ray binary properties of the objects. Using the 2MASS counterpart of well-known microquasars, we found that those with a high-mass nature follow a linear behavior in the infrared color-color diagram, while the low mass ones display a more random distribution. Based on this information, we suggest a possible region in the infrared diagram for new microquasar candidates (Fig 2). As a result, we found 3 objects that we propose as likely galactic high mass X-ray binaries and microquasar candidates (Fig 3).

\section{Conclusions and future prospects}

The methodology of database cross-identification has shown to be useful for proposing micro- 


\begin{tabular}{|c|c|c|c|c|c|c|c|c|c|}
\hline \multirow{2}{*}{$\begin{array}{l}\text { Src } \\
\#\end{array}$} & \multirow{2}{*}{$\begin{array}{l}\text { IAU name } \\
2 \mathrm{XMMp} \mathrm{J+}\end{array}$} & \multicolumn{2}{|c|}{ Coordinates J2000 } & \multicolumn{2}{|c|}{ Hardness ratio } & \multicolumn{3}{|c|}{ X-ray properties } & \multirow{2}{*}{$\begin{array}{l}\text { flag } \\
\text { Id. }\end{array}$} \\
\hline & & RA & DEC & $\mathrm{HR}_{x}$ & $\mathrm{HR}_{y}$ & $\mathrm{~N}_{\mathrm{H}}\left[10^{22}\right]$ & $\Gamma$ & Flux $\left[10^{-14}\right]$ & \\
\hline 15 & $035956.3+541855$ & $03: 59: 56.34$ & $54: 18: 55.71$ & 0.90 & 0.42 & 1.30 & 2.10 & 4.7 & $1^{\dagger}$ \\
\hline 44 & $165402.0-394630$ & $16: 54: 2.05$ & $-39: 46: 30.36$ & 0.61 & 0.85 & 0.22 & 0.00 & $8.52 \pm 1.1$ & $1^{\dagger}$ \\
\hline 64 & $183134.7-033538$ & $18: 31: 34.75$ & $-03: 35: 38.67$ & 0.21 & 0.69 & 0.05 & 0.50 & $2.52 \pm 0.9$ & $1^{\dagger+}$ \\
\hline
\end{tabular}

Figure 3: Proposed microquasar candidates obtained from our latest method of database cross-identification. Sources are presented in order of RA. The X-ray flux is in $\mathrm{ergs} \mathrm{s}^{-1} \mathrm{~cm}^{-2}$. It was computed in the SXSSC using an energy conversion factor (ECF) in the $0.2-12 \mathrm{keV}$ energy band. $\mathrm{N}_{H}$ is in units of $\mathrm{cm}^{-2}$. Flag-Id column refers to: (1)- unidentified; (E) - Source with 2MASS counterpart.

quasar candidates when using appropriate catalogues and selection criteria. Further improvements and refinements of this methodology are being developed for us to be ready when new catalogues, more sensitive and covering new regions of the sky, become available. Up to now, and mainly based on recent XMM-Newton catalogues, three new microquasar candidates are being proposed. In addition, thanks to the MAGPIS radio survey we have also identified the star LS 5007 as a another possible candidate. All these sources are currently being followed up in depth in order to find out the true origin of their radio and X-ray emission.

\section{References}

[1] J.A. Combi, J.F. Albacete-Colombo, J. Martí, A new search strategy for microquasar candidates using NVSS/2MASS and XMM-Newton data, A\&A 477, 1, 125 (2008) [arXiv:0710.5831].

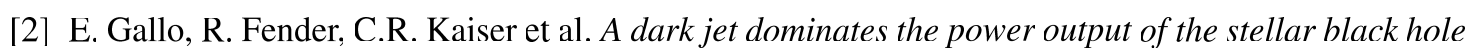
Cygnus X-1, Nature 436, 7052, 819 (2005). [arXiv:astro-ph/0508228].

[3] D.J. Helfand et al, MAGPIS: A Multi-Array Galactic Plane Imaging Survey, AJ 131,2525 (2006) [arXiv:astro-ph/0510468].

[4] C.R. Kaiser et al, Revision of the Properties of the GRS 1915+105.Jets: Clues from the Large-Scale Structure, AP.J 612, 1, 332 (2004) [arXiv:astro-ph/0405206].

[5] J. Martí, J.M. Paredes, M. Ribó, The system LS 5039: a new massive radio emitting X-ray binary, A\&A 338, L71 (1998).

[6] J.M. Paredes, J. Martí, M. Ribó et al., Discovery of a High-Energy Gamma-Ray-Emitting Persistent Microquasar, Science, 288, 2340 (2000) [arXiv:astro-ph/0102235].

17| J.M. Paredes, M. Ribó, J. Martí, A search for microquasar candidates at low galactic latitudes A\&A 394, 193 (2002) [arXiv:astro-ph/0401514].

[8] J.M. Paredes,Microquasars as High-energy Gamma-ray Sources, Ch.I.A\&A 5 Supplement, 121 (2004) [astro-ph/0409226].

[9] M. Ribó, J.M. Paredes, J. Martí et al., Results of a search for new microquasars in the Galaxy, $R M x A A$ 194, 23 (2004) [arXiv:astro-ph/0402054].

[10] C.B. Stephenson and N. Sanduleak, Luminous Stars in the Southern Milky Way. Publ. Warner \& Swasey Obs. 1,1 (1971).

[11] R.L. White, R.H. Becker, D.J. Helfand, New Catalogs of Compact Radio Sources in the Galactic Plane, AJ 130, 586 (2005). 